\title{
Effects of exergames on trunk balance control in paraplegic patients
}

\author{
Efeitos dos exergames no controle de tronco de pacientes paraplégicos
}

\author{
Simone de Paula (D), Kétlin Caroline Griebeler (D), Marta Rosecler Bez (D), \\ Claudio Felipe Kolling da Rocha (D)*
}

Universidade Feevale, Novo Hamburgo, RS, Brazil

\begin{abstract}
Introduction: Due to motivation and immediate feedback during activities, exergame-based physical therapy may improve trunk balance and functionality in individuals with spinal cord injury (SCI). Objective: evaluate the effects of exergames on the trunk control of paraplegics with spinal cord injury or meningomyelocele. Method: case series involving four paraplegic patients, with SCI or meningomyelocele. Participants underwent a rehabilitation protocol using the exergame Nintendo Wiiß, attending one weekly session for 4 weeks. Patients were encouraged to perform trunk movements in the sitting position using the Swordplay and Canoeing games. Data were collected before and after the intervention by applying the functional reach test adapted for trunk control evaluation, and transfer time and propulsion tests for functional assessment. Results: The intervention increased trunk control in $75 \%$ of the patients, with improvement varying between 6.4 and 25\%. In the propulsion test, the intervention led to a decrease in half of the patients. For the cadence variable, in the same test, there was a reduction in the number of propulsions in $75 \%$ of the cases. In the transfer test, the intervention led to reduced chair-to-bed transfer time in all patients. Conclusion: Rehabilitation of paraplegics with the use of exergames can be considered viable, innovative and effective. However, future research with greater methodological rigor should be conducted in order to analyze the clinical applicability of this approach.
\end{abstract}

Keywords: Physical Therapy. Spinal Cord Injuries. Paraplegia. Video Games. Meningomyelocele.

*DPS: PhD, email sdpaula@feevale.br GKC: BS, email: ketlincg@gmail.com BMR: PhD, email: martabez@feevale.br RCF: PhD, email: claudiodarocha@feevale.br 
Resumo

Introdução: Em virtude da motivação e do feedback imediato durante as atividades, os exergames durante a Fisioterapia podem favorecer o equilíbrio de tronco e a funcionalidade em lesados medulares. Objetivo: Avaliar os efeitos dos exergames no controle de tronco de paraplégicos. Método: Série de casos com quatro pacientes paraplégicos, portadores de lesão medular ou mielomeningocele. Os participantes foram submetidos a um protocolo de reabilitação com o uso do exergame Nintendo Wii ${ }^{\circledR}$, com frequência de uma sessão semanal durante 4 semanas. $O$ paciente foi estimulado a realizar movimentos de tronco na posição sentada através dos jogos esgrima e canoagem. Os instrumentos utilizados para a coleta de dados antes e depois da intervenção foram o Teste de Alcance Funcional adaptado para avaliação do controle de tronco, e o Tempo de Transferência e o Teste de Propulsão para a avaliação funcional. Resultados: A intervenção gerou um aumento absoluto do controle de tronco em $75 \%$ dos pacientes, com a melhora variando entre 6,4 e 25\%. No Teste de Propulsão, a intervenção levou à redução absoluta do tempo em metade dos pacientes. Para a variável cadência, no mesmo teste, houve redução do número de propulsões em $75 \%$ dos casos. No Teste de Transferências, a intervenção gerou redução do tempo entre a cadeira e a cama em todos os pacientes. Conclusão: A reabilitação de paraplégicos com o uso de exergames pode ser considerada viável, inovadora e efetiva. No entanto, pesquisas futuras com maior rigor metodológico devem ser realizadas com vistas à aplicabilidade clínica desta abordagem.

Palavras-chave: Fisioterapia. Traumatismos da Medula Espinal. Paraplegia. Jogos de Vídeo. Mielomeningocele.

\section{Introduction}

Spinal cord injury (SCI) is a condition with permanent debilitating clinical manifestations generated by partial or total impairment of spinal cord functions, caused by a lesion that affects the anatomic integrity of this structure. In light of their significant functional and socioeconomic impact, SCI sequelae are considered a public health problem in Brazil and worldwide. Recent data estimate that the world prevalence of this condition is between 236 and 1,298/million people, and around 180,000 individuals per year are affected by spinal cord damage [1].

Spinal cord injury can result in partial or total loss of motor and/or sensitive functions, thereby compromising the urinary, respiratory, circulatory and reproductive systems, significantly affecting mobility. The etiological factors for these disabilities may be related to traumatic events, including automobile accidents, gunshot wounds and diving in shallow water, and non-traumatic causes such as tumors, infections and congenital malformations [2, 3]. In relation to functional categories, SCIs are divided into tetraplegia and paraplegia. Tetraplegia is partial or total paralysis of the four limbs and trunk resulting from spinal cord injury, while paraplegia is partial or complete paralysis of the lower limbs and trunk due to thoracic, lumbar or sacral lesions [3].
In wheelchair-bound paraplegics, all normal functional activities depend on trunk control for movement. Thus, postural control and balance reactions in the sitting position should simultaneously provide stability and mobility in the activities of daily living (ADL), such as eating, dressing and wheelchair-to-bed transfers [4-6].

Given the essential role of trunk control and stability for the independence and autonomy of patients with SCIs, physiotherapy is an indispensable resource for the rehabilitation of these individuals. Using specific techniques, physiotherapists aim to stimulate neuromuscular components related to trunk control, thereby strengthening functional skills and social reintegration [7]. A number of therapeutic approaches have been used to optimize balance reactions in the sitting position, including functional electrical stimulation, task-based training and kinesiotherapy [8-10].

However, due to the lack of motivational elements and low patient adherence for comprehensive application of physiotherapy protocols, the use of technology has been gaining interest in healthcare and rehabilitation research. In this respect, the use of virtual games and virtual reality during physiotherapy enables a creative and motivating scenario where patients interact 
through kinesthetic, visual, tactile, auditory and/or sensory stimuli, aspects that favor satisfactory results, primarily in relation to the gait [11], balance [12] and upper limb functionality [13] of neurological patients. The main advantages of these approaches are the use of gratifying tasks with greater motivation to undergo treatment, immediate feedback during activities, information storage and considerable patient interactivity, combining rehabilitation and fun for all age groups, especially patients that need long-term treatment [14].

Associating technology with physical activity can be achieved in exergames, electronic games that require body movement and a combination of physical exercise and computer games [15]. In this type of game, real movements are converted to the virtual environment. The users actively engage in sports and physical exercise in a playful manner and interact with the equipment, which differs from traditional videogames, since they require physical exertion and motor capacities during the games [16]. Significant gains in motor skills were observed in children with developmental coordination disorders using exergames [17]. According to Barnett et al. [18] and Vernadakis et al. [19], the longer exergames are played, the greater the ability to control objects.

Despite recent favorable scientific evidence, studies on the use of virtual games in wheelchair-bound patients remain scarce. As such, the aim of the present study was to assess the effects of exergame-based rehabilitation on the trunk control of patients with spinal cord injury.

\section{Method}

\section{Design and sample}

This is a quantitative study using a case series of four wheelchair-bound paraplegic patients diagnosed with traumatic or congenital SCI, average age of $35.0 \pm 29.2$ years, recruited at a physiotherapy school clinic in Southern Brazil. All participants provided written informed consent. The study was approved by the Research Ethics Committee (CAAE 52077315.8.0000.5348) and complies with the guidelines of Resolution no. 466/12 of the National Health Council, which regulates research involving human beings.
Intervention

The participants selected were submitted to a rehabilitation protocol using the Nintendo $\mathrm{Wii}^{\circledR}$ exergame, consisting of weekly 20-minute sessions over a 4-week period. Using the WiiSports ${ }^{\circledR}$ games platform, the patients were encouraged to perform rotation movements and anterior and lateral trunk inclination associated with the upper limbs. Despite being commercial and not intended for use in rehabilitation, these games are easy to understand and enable movements with the trunk and upper limbs.

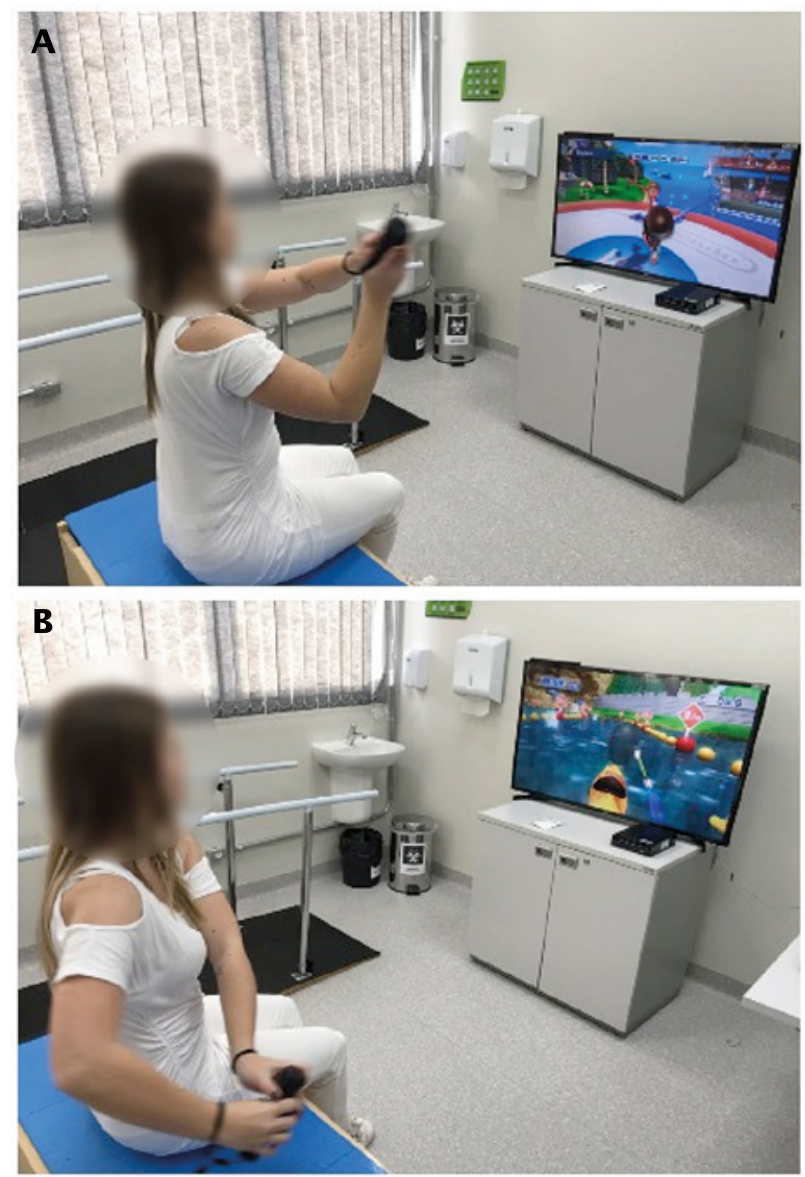

Figure 1 - Intervention using the Nintendo Wii ${ }^{\circledR}$ with Swordplay (A) and Canoeing (B) games.

The Nintendo $\mathrm{Wii}^{\circledR}$ is an easy-to-use game console with a wireless controller (Wii Remote ${ }^{\circledR}$ ), equipped with a three-axis accelerometer for detecting hand motions. This control has a sensor that can quantify player movement, engaging them more in the game 
with auditory and visual feedback. In addition, the control has a vibration system and speaker that emits sounds near the player.

In the present study, patients performed the exercises while sitting, with hips, knees and ankles at $90^{\circ}$, in a standardized seat, with feet on a rubber step and facing the game screen (Figure 1 ) at a standard distance of 1 meter. In order to assess kinetic-functional outcomes, the instruments used for data collection were transfer time and the propulsion test to evaluate functionality in a wheelchair, and the functional reach test, adapted to analyze trunk control. Assessments were conducted before and 4 weeks after the intervention.

During the exergame protocol, patients underwent conventional physiotherapy once a week, consisting of global stretching and passive leg exercises.

Functional and clinical data

The clinical data of participants were collected from the physiotherapy clinic records. In order to identify the level of functional independence in activities of daily living (ADL), participants were classified into three categories: independent, where all basic activities of daily living are executed independently; semi-independent, representing the impairment of at least one of the functions influenced by culture and learning (bathing and/or dressing and/or using the bathroom); and complete dependence, that is, one of the simple vegetative functions compromised (transfer and/or continence), in addition to being dependent for bathing, dressing and using the bathroom [20].

\section{Adapted Functional Reach Test}

The adapted functional reach test was used to assess sitting trunk control, following the method described by Lynck et al. [21]. For this analysis, participants remained seated in their wheelchairs, with no arm support and hips, knees and ankles at $90^{\circ}$ flexion. A tape measure was mounted on the wall, parallel to the floor, at the shoulder height of the participant. The subjects were asked to flex their shoulder to $90^{\circ}$ and close their fist. Next, they were instructed to lean forward as far as possible, without losing their balance or moving the wheelchair. Displacement was measured on the tape measure, using the average of three functional reach attempts.
Wheelchair propulsion test

The wheelchair propulsion test determines patient mobility in a wheelchair. To that end, the patients were asked to move $10 \mathrm{~m}$ in their wheelchair on a smooth even surface, from a stationary starting point. Observation and a stopwatch were used to measure displacement time (in seconds) and count the number of cadences or propulsion frequency. Cadence (cycles/s) was defined as the first contact of the patient's hands with the upper edge of the wheelchair's rear wheel until the end of propulsion. The average of three attempts (30 seconds apart) was considered for data analysis [22].

\section{Transfer test}

Since this is a case series, data analysis was carried out to compare between assessments before and after the exergame-based rehabilitation protocol, and the results graphically presented. The graphs show the percentage of absolute improvement for each patient in the variables analyzed. Positive percentages represent improvement and their negative counterparts a decline.

\section{Statistical analysis}

Since this is a case series, data analysis was carried out to compare between assessments before and after the exergame-based rehabilitation protocol, and the results graphically presented. The graphs show the percentage of absolute improvement for each patient in the variables analyzed. Positive percentages represent improvement and their negative counterparts a decline.

\section{Results}

The sample was composed of two patients diagnosed with high lumbar level meningomyelocele, one with L1 and the other T11 level traumatic spinal cord injury. The wide age variation reflects the population treated at the clinic, patients 1, 2, 3 and 4 aged 16, 5, 52 and 67 years, respectively. The functional independence level varied from independence to incomplete dependence, which remained unchanged after the 4-week exergame intervention (Table 1). 
Table 1 - Clinical-functional characteristics of the case studies

\begin{tabular}{cccc}
\hline Case & Age (years) & Clinical diagnosis & Level of functional independence \\
\hline 1 & 16 & High lumbar level meningomyelocele & Incomplete dependence \\
2 & 5 & High lumbar level meningomyelocele & Incomplete dependence \\
3 & 52 & Traumatic spinal cord injury (T11) & Semi-dependence \\
4 & 67 & Traumatic spinal cord injury (L1) & Independence
\end{tabular}

In relation to trunk control, intervention increased adapted functional reach in $75 \%$ of the patients, with improvements varying between 6.4 and $25 \%$, except for case 1 . The results are graphically presented in Figure 2.

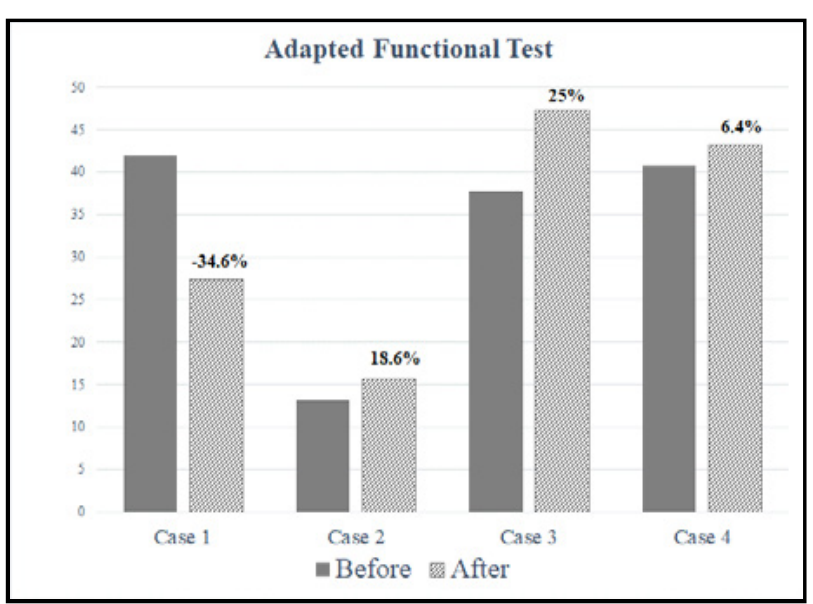

Figure 2 - Results of the adapted functional reach test before and after intervention with the Nintendo $\mathrm{Wil}^{\circledR}$. Note: The percentage improvement is shown for each patient.

In the propulsion test, the intervention caused an absolute decline in time in half the patients, ranging between 3.8 and $7.8 \%$ improvement. Case 3 showed positive post-intervention effects, and once again, case 1 exhibited the worst performance in the test (Figure 3A). For the variable cadence, in the same test, the number of propulsions declined in $75 \%$ of the cases, with an improvement between 7.7 and $11 \%$ The intervention had no effect on cadence for case 1 (Figure 3B).

In the task test, chair-to-bed transfer time and vice versa were assessed. The intervention caused a decrease in the former in $100 \%$ of the patients, resulting in improvements between 9.7 and 37\% (Figure 4A). In relation to bed-to-chair transfer time, there was also an improvement in $100 \%$ of the patients, with lower times between 14.3 and 52.6\% (Figure 4B).
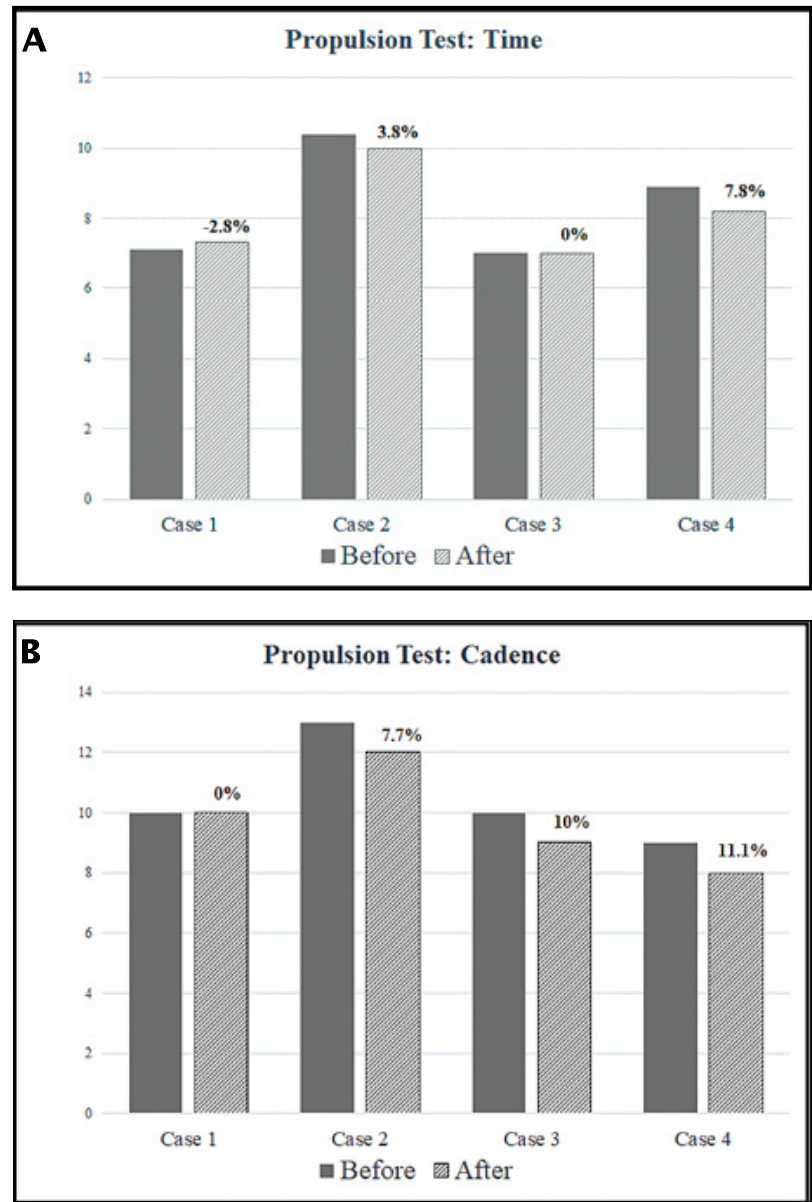

Figure 3 - Propulsion test results before and after intervention with the Nintendo $\mathrm{Wii}^{\circledR}$. A. Displacement time; $\mathrm{B}$. Number of propulsions.

Note: The percentage improvement is shown for each patient. 

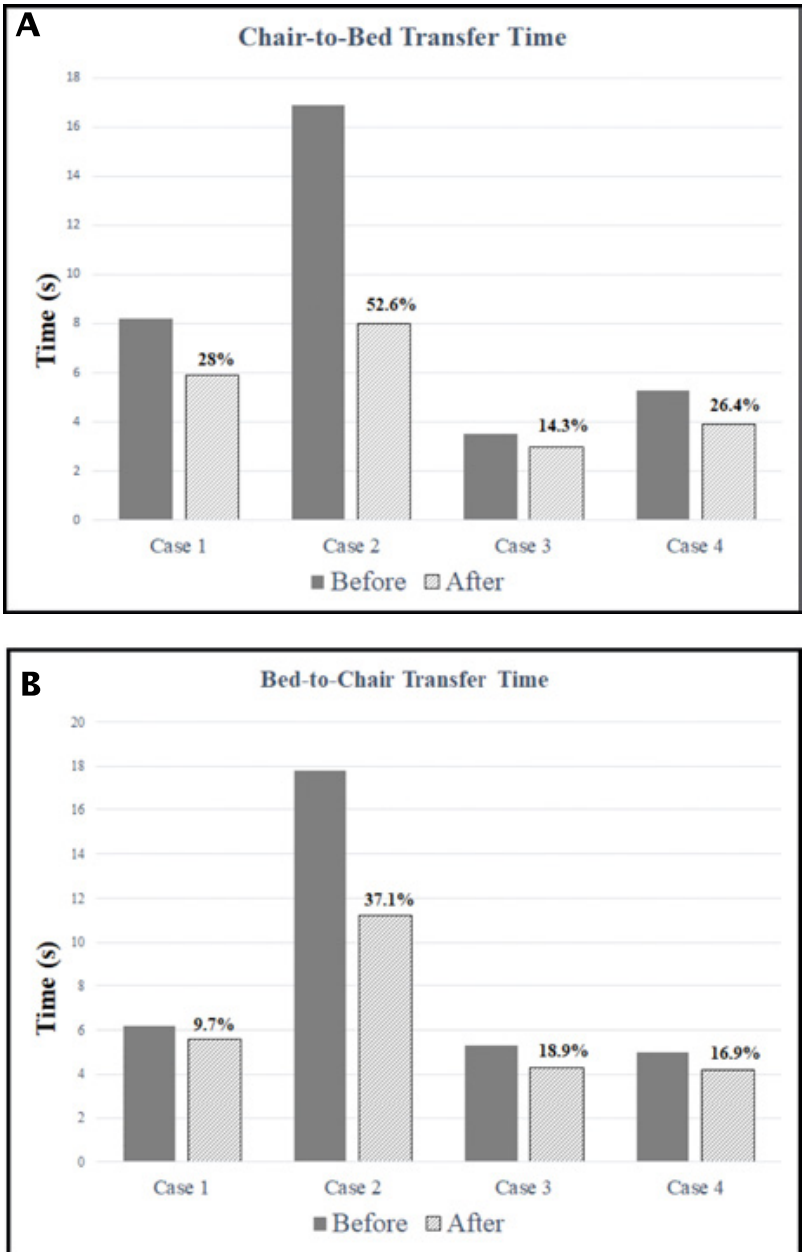

Figure 4 - Results of the task test before and after intervention with the Nintendo Wii ${ }^{\circledR}$. A. Chair-to-bed transfer time; B. Bed-to-chair transfer time.

Note: The percentage improvement is shown for each patient.

\section{Discussion}

The functional activities of paraplegic patients depend on trunk control for movement. This skill plays an essential role in balance, transfers and the use of upper limbs in wheelchair-bound patients, simultaneously providing stability and mobility so that individuals can perform their daily activities [23].

In the present study, exergame-based rehabilitation was used as a resource to optimize sitting balance reactions, emphasizing trunk control. After the intervention, most participants obtained an increase in the adapted functional reach test, indicating satisfactory effects on the ability to lean forward, an essential task to perform ADL. According to Gagnon et al. [24], impaired trunk balance reduces wheelchair propulsion and interferes in the performance of other activities, such as the ability to transfer from a chair to the bed. In light of these aspects, the effects of exergames on wheelchair propulsion and chair-to-bed transfer were also analyzed. These variables demonstrated that the patients exhibited favorable outcomes, suggesting that, in addition to the positive improvement in trunk control, using Nintendo $\mathrm{Wii}^{\circledR}$ during rehabilitation favored the development of functional skills.

Similar to our study, the results of a randomized clinical trial conducted by Tak et al. [25] in patients with spinal cord injury showed that paraplegics who underwent a 6-week virtual rehabilitation protocol obtained a significant increase in static and dynamic sitting balance when compared to their control group counterparts. The authors attributed the positive findings of the study to the visual feedback provided by the virtual scenario. In addition, Wall et al. [26] demonstrated that after 7 weeks of training with the Nintendo $\mathrm{Wii}^{\circledR}$, paraplegic patients improved their performance in the Functional Reach Test. Moreover, after 4 weeks' follow-up, the participants also performed well in the variables analyzed, indicating that improved balance persisted even after the end of the exergame rehabilitation protocol. In their case study of a patient diagnosed with meningomyelocele, Betker et al. [25] observed a substantial rise in sitting dynamic control, in addition to significant increases in attention and motivation during therapy.

Monteiro Junior and Silva [27] reported that the amount of sensory information provided by virtual rehabilitation systems seems to be a strong element in acquiring balance and better functionality. Despite the methodological heterogeneity, this systematic review found that in most studies, the visual feedback of the virtual game was an effective resource for individuals with neurological diseases to recover their balance and motor skills.

Balance is the early and momentary identification of postural instabilities by the nervous system, which generates coordinated responses and adjustments that allow individuals to return to their support base, thereby avoiding falls [28]. Sigrist et al. [29] added that the development of balance reactions involves the integration of different afferent information to perform specific tasks, including vision, the vestibular system and proprioception. In this respect, strategies that use virtual games have proven to be an effective resource for optimizing motor learning, providing immediate sensory-motor feedback of movement changes. Moreover, during exergame exercises, the user does not focus on the movement itself, but 
rather on the outcome of the movement. This is an important characteristic for rehabilitation programs, since, during ADL, the patient focuses on the task and not on maintaining balance or executing the movement. Thus, exergames may reduce anxiety and the fear of falling by using safe virtual scenarios, thereby motivating patients to engage in their treatment [30].

In addition to balance, it is important to consider that trunk stability is also dependent on musculoskeletal components. In individuals with SCI, dynamic and static instability to recruit the straight, transverse abdominal, quadratus lumborum and erector spinae muscles may lead to compensatory strategies using non-postural muscles such as the latissimus dorsi, trapezius and pectoralis major. As such, paraplegic patients develop new postural control processes to maintain stability while sitting, which may result in posterior pelvic tilt and an increase in thoracolumbar kyphoscoliosis [4].

Patient motivation during the rehabilitation process is not totally understood. However, this aspect has been considered an important adjuvant for treatment. Thornton et al. [31] indicates that patients who participate in balance training programs based on digital resources express greater satisfaction, more confidence in their functional abilities, and higher self-esteem than those who take part in conventional programs. Butler and Willett [32] also reported that exergames allow patients to focus on the activities, and may be a more motivating therapeutic strategy than tedious conventional exercises. The authors also state that rehabilitation using games enables patients to overcome challenges and obtain better game results, which leads to greater cognitive involvement.

In addition to motivation, the movements needed to induce neuroplasticity mechanisms are a determining factor for successful rehabilitation. In this respect, positive emotional impactand having fun during digital gamebased therapy may increase the number of movements during physiotherapy and optimize motor learning [33].

Injured neurons and axons can grow in denerved regions in order to reconstruct neuronal circuits to compensate for compromised sensorimotor functions [34]. This phenomenon is optimized in the presence of some type of extrinsic feedback such as vision. Visual information provided by virtual reality seems to be closely involved in the functional recovery of individuals with neurological impairment, and is primarily responsible for improved motor control. Given that this therapy requires weekly systematized movements, authors have hypothesized that important chronic neural adaptations occur as a function of this treatment, such as an increase in neurotrophic factors (BDNF, VEGF and IGF-1), which play an essential role in chemical signaling for neurogenesis and angiogenesis and may enhance cognitive and motor performance [35].

Due to the small sample and heterogeneity of the cases assessed, the results obtained cannot be generalized. In addition, the duration of the intervention (4 weeks) and absence of follow-up does not provide enough information about the functional impacts of long-term virtual games. Finally, it is important to underscore that the Nintendo $\mathrm{Wii}^{\circledR}$ is a game console developed for individuals with no neuromotor disorders. Thus, randomized controlled trials should be conducted in order to obtain more consistent results for physiotherapy clinical applicability based on exergames, considering specific therapeutic plans for neurological patients.

\section{Conclusion}

Despite the small sample and short intervention time, the paraplegic patients in the present study improved their trunk balance and functional activities in a wheelchair. These findings suggest that exergames are a viable, innovative and effective physiotherapy resource for rehabilitating patients with neuromotor disabilities.

\section{Acknowledgement}

The authors thank Feevale University for its support in developing this line of research.

\section{References}

1. Quadri SA, Farooqui M, Ikram A, Zafar A, Khan MA, Suriya SS, et al. Recent update on basic mechanisms of spinal cord injury. Neurosurg Rev. 2020;43(2):425-41.

2. Lee BB, Cripps RA, Fitzharris M, Wing PC. The global map for traumatic spinal cord injury epidemiology: update 2011, global incidence rate. Spinal Cord. 2014;52(2):110-6. 
3. Stokes M, Stack E. Physical management for neurological conditions. Amsterdam: Churchill Livingstone; 2013.

4. Milosevic M, Yokoyama H, Grangeon M, Masani K, Popovic MR, Nakazawa $\mathrm{K}$, et al. Muscle synergies reveal impaired trunk muscle coordination strategies in individuals with thoracic spinal cord injury. J Electromyogr Kinesiol. 2017;36:40-8.

5. Bjerkefors A, Carpenter MG, Cresswell AG, Thorstensson A. Trunk muscle activation in a person with clinically complete thoracic spinal cord injury. J Rehabil Med. 2009;41(5):390-2.

6. Gabison S, Verrier MC, Nadeau S, Gagnon DH, Roy A, Flett HM. Trunk strength and function using the multidirectional reach distance in individuals with non-traumatic spinal cord injury. J Spinal Cord Med. 2014;37(5):537-47.

7. Harvey LA. Physiotherapy rehabilitation for people with spinal cord injuries. J Physiother. 2016;62(1):4-11.

8. Cabanas-Valdeés R, Bagur-Calafat C, Girabent-Farrés M, Caballero-Gómez FM, Hernaández-Valiño M, Urrútia Cuchí $\mathrm{G}$. The effect of additional core stability exercises on improving dynamic sitting balance and trunk control for subacute stroke patients: a randomized controlled trial. Clin Rehabil. 2016;30(10):1024-33.

9. Vanoncini M, Holderbaum WJ, Andrews B. Electrical Stimulation for trunk control in paraplegia: A feasibility study. Control Eng Pract. 2012;20(12):1247-58.

10. Tse CM, Chisholm AE, Lam T, Eng JJ, SCIRE Research Team. A systematic review of the effectiveness of taskspecific rehabilitation interventions for improving independent sitting and standing function in spinal cord injury. J Spinal Cord Med. 2018;41(3):254-66.

11. Fung J, Richards CL, Malouin F, McFadyen BJ, Lamontagne A. A treadmill and motion coupled virtual reality system for gait training post-stroke. Cyberpsychol Behav. 2006;9(2):157-62.

12. Morone G, Tramontano $\mathrm{M}$, Iosa $\mathrm{M}$, Shofany J, Iemma A, Musicco M, et al. The efficacy of balance training with video game-based therapy in subacute stroke patients: a randomized controlled trial. Biomed Res Int. 2014;2014:580861.
13. Zoccolillo L, Morelli D, Cincotti F, Muzzioli L, Gobbetti T, Paolucci S, et al. Video-game based therapy performed by children with cerebral palsy: a cross-over randomized controlled trial and a cross-sectional quantitative measure of physical activity. Eur J Phys Rehabil Med. 2015;51(6):669-76.

14. Sveistrup H. Motor rehabilitation using virtual reality. J Neuroeng Rehabil. 2004;1(1):10.

15. Lin JH. "Just Dance": The Effects of Exergame Feedback and Controller Use on Physical Activity and Psychological Outcomes. Games Health J. 2015;4(3):183-9.

16. Hammond J, Jones V, Hill EL, Green D, Male I. An investigation of the impact of regular use of the Wii Fit to improve motor and psychosocial outcomes in children with movement difficulties: a pilot study. Child Care Health Dev. 2014;40(2):165-75.

17. Deutsch JE, Brettler A, Smith C, Welsh J, John R, GuarreraBowlby P, et al. Nintendo Wii Sports and Wii Fit game analysis, validation, and application to stroke rehabilitation. Top Stroke Rehabil. 2011;18(6):701-19.

18. Barnett LM, Hinkley T, Okely AD, Hesketh K, Salmon J. Use of electronic games by young children and fundamental movement skills? Percept Mot Skills. 2012;114(3):1023-34.

19. Vernadakis N, Papastergiou M, Zetou E, Antoniou P. The impact of an exergame-based intervention on children's fundamental motor skills. Comput Educ. 2015;83:90-102.

20. Moraes EN, Pereira AMVB, Azevedo RS, Moraes FL. Avaliação multidimensional do idoso. Curitiba: SESA; 2018.

21. Lynch SM, Leahy P, Barker SP. Reliability of measurements obtained with a modified functional reach test in subjects with spinal cord injury. Phys Ther. 1998;78(2):128-33.

22. Askari S, Kirby RL, Parker K, Thompson K, O'Neill J. Wheelchair propulsion test: development and measurement properties of a new test for manual wheelchair users. Arch Phys Med Rehabil. 2013;94(9):1690-8.

23. Chen CL, Yeung KT, Bih LI, Wang CH, Chen MI, Chien JC. The relationship between sitting stability and functional performance in patients with paraplegia. Arch Phys Med Rehabil. 2003;84(9):1276-81. 
24. Gagnon D, Verrier M, Masani K, Nadeau S, Aissaoui R, Popovic M. Effects of trunk impairments on manual wheelchair propulsion among individuals with a spinal cord injury: a brief overview and future challenges. Top Spinal Cord Inj Rehabil. 2009;15(2):59-70.

25. Tak S, Choi W, Lee S. Game-based virtual reality training improves sitting balance after spinal cord injury: a single-blinded, randomized controlled trial. Med Sci Tech. 2015;56:53-9.

26. Wall T, Feinn R, Chui K, Cheng MS. The effects of the Nintendo Wii Fit on gait, balance, and quality of life in individuals with incomplete spinal cord injury. J Spinal Cord Med. 2015;38(6):777-83.

27. Monteiro Jr RS, Silva EB. Efetividade da reabilitação virtual no equilíbrio corporal e habilidades motoras de indivíduos com déficit neuromotor: uma revisão sistemática. Rev Bras Ativ Fis e Saude. 2012;17(3):224-30.

28. Betker AL, Szturm T, Moussavi ZK, Nett C. Video gamebased exercises for balance rehabilitation: a single-subject design. Arch Phys Med Rehabil. 2006;87(8):1141-9.

29. Sigrist R, Rauter G, Riener R, Wolf P. Augmented visual, auditory, haptic, and multimodal feedback in motor learning: a review. Psychon Bull Rev. 2013;20(1):21-53.
30. Lelard T, Ahmaidi S. Effects of physical training on agerelated balance and postural control. Neurophysiol Clin. 2015;45(4-5):357-69.

31. Thornton M, Marshall S, McComas J, Finestone H, McCormick A, Sveistrup H. Benefits of activity and virtual reality based balance exercise programmes for adults with traumatic brain injury: perceptions of participants and their caregivers. Brain Inj. 2005;19(12):989-1000.

32. Butler DP, Willett K. Wii-habilitation: is there a role in trauma? Injury. 2010;41(9):883-5.

33. Lohse K, Shirzad N, Verster A, Hodges N, van der Loos HF. Video games and rehabilitation: using design principles to enhance engagement in physical therapy. J Neurol Phys Ther. 2013;37(4):166-75.

34. Liu J, Yang X, Jiang L, Wang C, Yang M. Neural plasticity after spinal cord injury. Neural Regen Res. 2012;7(5):386-91.

35. Jang SH, You SH, Hallett M, Cho YW, Park CM, Cho SH, et al. Cortical reorganization and associated functional motor recovery after virtual reality in patients with chronic stroke: an experimenter-blind preliminary study. Arch Phys Med Rehabil. 2005;86(11):2218-23.

Received: 10/08/2018

Recebido: 08/10/2018

Approved: 02/03/2020

Aprovado: 03/02/2020 